\title{
Utilização de funções matemáticas no estudo da curva de lactação em caprinos $^{1}$
}

\section{Vinícius Pereira Guimarães ${ }^{2}$, Marcelo Teixeira Rodrigues ${ }^{3}$, José Lindenberg Rocha Sarmento ${ }^{4}$, Denis Teixeira da Rocha ${ }^{5}$}

\author{
1 Parte da dissertação apresentada à UFV pelo primeiro autor para obtenção do título de Magister Scientiae em Zootecnia. \\ 2 Pós-graduação em Zootecnia - UFV. \\ ${ }^{3}$ Departamento de Zootecnia - UFV. \\ 4 Pós-graduação em Genética e Melhoramento - UFV. \\ ${ }^{5}$ Graduação em Zootecnia - UFV.
}

RESUMO - Objetivou-se com este estudo comparar funções matemáticas propostas na literatura para o ajuste da curva de lactação em caprinos leiteiros. Foram utilizados 23.502 controles leiteiros, totalizando 748 lactações de cabras das raças Saanen e Alpina. A estimativa dos parâmetros foi feita por meio de regressões não-lineares, pelo procedimento NLIN do programa SAS (Statistical Analysis System), utilizando-se o método modificado de Gauss-Newton. Para a escolha do melhor modelo, utilizou-se o gráfico de distribuição de resíduo, a média dos desvios absolutos, o quadrado médio dos resíduos e a comparação das curvas estimada e observada por meio de avaliação visual. Foram testadas a igualdade de parâmetros e a identidade de modelos de regressão não-linear para verificar se os parâmetros das curvas para cada ordem de lactação e para cada raça eram iguais. Entre os modelos estudados, o proposto por Nelder (1966), $Y=t / a+b t+c t^{2}$, foi o que apresentou o melhor ajuste para a curva de lactação dos animais. Houve diferenças entre os parâmetros do modelo escolhido para as diferentes lactações e raças; mesmo assim, considerou-se na análise uma única equação para facilitar o estudo.

Palavras-chave: cabras leiteiras, modelos matemáticos, produção de leite

\section{Use of mathematical functions in the study of lactation curves in goats}

ABSTRACT - Production of low cost milk is the most relevant aspect in dairy goat systems. Therefore, studying the behavior of the lactation curve of dairy goats is important to implement animal breeding programs as well as for plan and introduce new management tools. The objective of this study was to select among mathematical models published in the literature the one that best describe the lactation curve of dairy goats. A dataset constituted of 23,502 observations of milk yield from 748 lactations of Saanen and Brown Alpine (Oberhaslis) goats was used. Estimation of the parameters was done by the non-linear regression procedure of SAS using the modified method of Gauss-Newton. Residual plot, absolute mean deviation, correlation coefficient and visual appraisal of predicted versus measured lactation curve all were used to determine the best model. The equality of parameters and the models identity of non-linear regression were used to test if the parameters of the lactation curve were similar for breed and order of lactation. Among the evaluated models, that proposed by Nelder (1966), $Y=t / a+b t+c t^{2}$, was the best compromise for adjustment of the lactation curve of goats. Even though differences among parameters of the chosen model for different lactation and breed were observed, only one equation was used in the analysis.

Key Words: dairy goats, mathematical models, milk production

\section{Introdução}

A redução do tamanho das propriedades rurais, em especial aquelas próximas aos centros urbanos e mão-deobra disponível nas zonas rurais e o custo da terra e dos insumos têm provocado a implantação de atividades agropecuárias intensivas visando maximizar o uso da terra.

A caprinocultura leiteira tem sido considerada uma alternativa viável para essas condições, pois possibilita a criação intensiva, a maximização do uso do solo e a facilidade de manuseio dos animais.

$\mathrm{Na}$ caprinocultura leiteira, a produção econômica do leite é a característica de maior relevância. O conhecimento do perfil e da magnitude da curva de lactação tem importante papel no melhoramento genético de caprinos, além de oferecer subsídios para o planejamento da atividade. Existem inúmeras alternativas para o conhecimento e a utilização da curva de lactação de um rebanho; uma delasé o estabelecimento 
de um programa de melhoramento baseado nas produções total e parcial dos animais, no qual seriam avaliados aspectos biológicos e econômicos da criação.

A curva de lactação também pode ser utilizada para o estudo da produção de leite individual ou de um rebanho e para obtenção de medidas de composição do leite ajustadas para o estágio de lactação (Fuertes et al., 1998). Esse conhecimento permite predizer a produção total por meio de uma informação de produção (Gipson \& Grossman, 1989). Assim, o produtor pode tomar decisões baseadas em produções no início da lactação, além de identificar problemas como mastite subclínica, quando ocorre abrupta redução na produção. Outra vantagem é a possibilidade de melhorar o manejo reprodutivo dos animais em lactação (Wood, 1980; Mcmanus et al., 1997), uma vez que podem ser estipuladas épocas de acasalamento para aumentar a produção utilizando informações do pico de produção.

$\mathrm{Na}$ literatura, são encontrados estudos comparando modelos estatísticos para o ajustamento de curvas de lactação. Diversos autores têm estudado essas funções, principalmente em bovinos (Cobby \& Le Du, 1978; Shanks et al., 1981; Papajcsik \& Bodero, 1988; Sherchand et al., 1995; Freitas et al., 1997; Bueno et al., 1998; Oliveira, 1999; Cobuci et al., 2000; Vargas et al., 2000).

A finalidade do modelo é prever a produção de leite da cabra em qualquer dia da lactação com o mínimo de erro possível, considerando também as variações ambientais (Fernández et al., 2002; Papajcsik \& Bodero, 1988). Os resultados deste estudo podem ser usados em modelos bioeconômicos, para determinação de estratégias de acasalamento tanto para cabras quanto para vacas (Vargas et al., 2000).

Desta forma, modelos empíricos têm sido utilizados para simulação da lactação e para previsão de futuras produções (Gipson \& Grossman, 1989), fazendo-se necessário avaliar quais modelos podem realmente fornecer melhor qualidade de informação para o ajustamento das curvas de lactação.

O modelo mais comumente utilizado para a estimativa de curvas de lactação é a função gama incompleta, proposta por Wood (1967), que tem proporcionado bom ajuste das curvas de lactação. Contudo, muitas variações que acontecem com a utilização desta função decorrem de problemas biológicos como, por exemplo, uma queda abrupta da produção depois do pico, decorrente de nutrição inadequada (Casoli et al.,1989). Então, mesmo com a ampla utilização da função gama incompleta, ocorrem desvios e falhas nas estimativas dos parâmetros da curva e na definição de seu formato.

Além dos problemas de estimação deste modelo, muitas variações podem ser atribuídas à espécie e às raças, que podem apresentar diferentes potenciais de produção. Portanto, torna-se fundamental o estudo de outros modelos que venham a caracterizar melhor essas diferenças, a fim de proporcionar adequado ajuste da curva.

Este estudo foi realizado com o objetivo de comparar funções matemáticas para o ajuste da curva de lactação em caprinos leiteiros.

\section{Material e Métodos}

Os dados utilizados neste estudo foram provenientes do rebanho do Setor de Caprinocultura da Universidade Federal de Viçosa, Viçosa - MG.

Os animais foram mantidos confinados em baias coletivas sob o sistema de estabulação livre (free stall), efetuando-se semanalmente o controle leiteiro durante o período de 2000 a 2003.

No banco inicial de dados, havia 41.562 registros de produção de leite. Na consistência dos dados, foram excluídas lactações em que o primeiro controle foi efetuado somente em tempo igual ou superior a 35 dias após o parto e intervalos sem controle de leite de, no máximo, 21 dias. Após as restrições, foram utilizados 23.502 registros de produção de leite para o estudo dos modelos no ajuste da curva de lactação, totalizando 748 lactações, pertencentes a 134 cabras Saanen e 309 Alpinas. Optou-se por estudar apenas até a quarta lactação, em razão do pequeno volume de informações existentes para as demais.

Para o ajuste das curvas de lactação, foram empregados 12 modelos (Tabela 1 ), nos quais $Y$ representa a produção de leite; $a, b, c$ e $d$ são parâmetros a serem estimados; e $t$ é o período de tempo (em semanas) após o parto em que foi medida a produção de leite.

Embora os símbolos utilizados para representar os parâmetros das funções sejam os mesmos, eles não possuem a mesma interpretação matemática ou biológica. Assim, nos modelos sugeridos, existem variações quanto ao significado de seus parâmetros e da representação de informações sobre o pico de produção e o tempo do pico.

O modelo de Brody et al. (1924) estima a produção inicial como sendo zero, com pico de produção em (c-b) $)^{-1} \ln (\mathrm{c} / \mathrm{b})$ dias depois do parto e posterior declínio.

Sikka (1950) propôs um modelo que descreve uma curva de lactação em forma de sino, em que o pico de produção estimado ocorre em b/2c, caracterizando o meio da distribuição.

Segundo Nelder (1966), o tempo para se atingir o pico de produção é estimado por $(\mathrm{a} / \mathrm{c})^{0,5}$, enquanto o valor da produção de leite no pico pode ser encontrado por $1 /\left[2(\mathrm{ac})^{0,5}+\mathrm{b}\right]$. 
Tabela 1 - Modelos utilizados no ajuste da curva de lactação Table 1 - Models used to fit the lactation curve

\begin{tabular}{|c|c|c|}
\hline & $\begin{array}{l}\text { Modelo } \\
\text { Model }\end{array}$ & $\begin{array}{l}\text { Autor } \\
\text { Author }\end{array}$ \\
\hline 1 & $Y=a e^{b t}-a e^{-c t}$ & Brody et al. (1924) \\
\hline 2 & $Y=a e^{\left(b t-c t^{2}\right)}$ & Sikka (1950) \\
\hline 3 & $Y=t / a+b t+c t^{2}$ & Nelder (1966) \\
\hline 4 & $Y=a t^{b} e^{-c t}$ & Wood (1967) \\
\hline 5 & $Y=\ln (a)+b \ln (t)-c t$ & Wood $(1967)^{*}$ \\
\hline 6 & $Y=a+b t-c t^{2}$ & Dave (1971) \\
\hline 7 & $Y=a-b t-a e^{-c t}$ & Cobby \& Le Du (1978) \\
\hline 8 & $Y=a-b t+c \ln (t)$ & Singh \& Gopal (1982) \\
\hline 9 & $Y=a t^{b c} e^{-c t}$ & Dhanoa (1981) \\
\hline 10 & $Y=a t e^{-c t}$ & Papajcsik \& Bodero (1988) \\
\hline 11 & $Y=a e^{\left(b t^{\prime}-c t^{\prime}+d / t\right)}$ & $\begin{array}{l}\text { Morant \& Gnanasakthy } \\
(1989)^{* *}\end{array}$ \\
\hline 12 & $Y=a t^{b e(-c t)}$ & $\begin{array}{l}\text { Cappio-Borlino et al. } \\
\text { (1995) }\end{array}$ \\
\hline
\end{tabular}

Y é a produção de leite; a, b, c e d são parâmetros da curva de lactação. $Y$ is the milk yield, $a, b, c$ and $d$ are parameters of the lactation curve.

* linearizado (linearized).

$* * \mathrm{t}^{\prime}=(\mathrm{t}-150) / 100$.

Pelo modelo de Wood (1967), tem-se que $Y$ é a produção de leite na semana $t$; $a$ é o parâmetro relacionado à produção inicial de leite; $b$ define a taxa de ascensão média na fase prépico de produção; e $c$ indica a taxa média de declínio da produção após o pico. Neste modelo, a persistência é definida como c- $(b+1)$, o tempo para o pico de produção, como b/c dias e a produção no pico, como a(b/c) $)^{b} e^{-b}$.

O modelo de Dave (1971) é uma equação quadrática em que o tempo para se atingir o pico é b/2c.

No modelo de Cobby \& Le Du (1978), os parâmetros $b$ e $c$ estão relacionados à persistência da lactação e ao declínio na produção, respectivamente, sendo o pico de produção estimado $\mathrm{em}^{-1} \ln (\mathrm{ac} / \mathrm{b})$ dias após o parto e o tempo para atingir este pico em $\ln (\mathrm{ac} / \mathrm{b})$, com posterior declínio.

O modelo de Singh \& Gopal (1982) não pode ser definido no tempo zero pela característica logarítimica da função, sendo o pico de produção predito de $\mathrm{c} / \mathrm{b}$ dias depois do parto seguido por um lento e contínuo declínio.

Dhanoa (1981) elaborou um modelo que foi uma reparametrização da função de Wood, sendo o parâmetro $b$ o tempo para se atingir o pico de produção.

Papajacsik \& Bodero (1988) descreveram um modelo semelhante ao de Wood, mas consideraram o valor do parâmetro $b$ como 1 , eliminando sua influência sobre o modelo.

O modelo de Morant \& Gnanasakthy (1989) assume que as mudanças na produção de leite após o pico não são constantes, como descrito pela redução exponencial, e assume uma transformação do tempo de produção de leite, como descrito na Tabela 1.

Cappio-Borlino et al. (1995) também fizeram uma modificação não-linear da função de Wood, indicada para produções com declínio acentuado logo após o pico da lactação e em que o pico ocorre em a(b/c) $)^{b c(-b)}$

As estimativas dos parâmetros foram feitas pelo processo iterativo pelo método de Gauss-Newton, utilizando-se o procedimento NLIN (SAS, 1999). Os modelos foram avaliados primeiramente para todas as lactações e depois para as lactações individuais. Posteriormente, foi feita uma análise para cada uma das quatro ordens de lactação e para cada uma das duas raças com o modelo escolhido.

Para a escolha do melhor modelo, utilizou-se o gráfico de distribuição de resíduo, a média dos desvios absolutos (MDA), o quadrado médio do resíduo (QMR) e a comparação das curvas estimada e observada por avaliação visual. O QMR foi corrigido dividindo-se a soma de quadrados do resíduo, obtida pela análise de variância, pelo número de observações, em uma estimativa de máxima verossimilhança. Esse procedimento foi feito para que se pudesse comparar o QMR dos diferentes modelos, em razão da existência de um modelo com número diferente de parâmetros (Sarmento et al., 2003).

Os modelos foram também comparados por meio do coeficiente de determinação corrigido para o número de parâmetros do modelo. O coeficiente corrigido foi utilizado pelo fato de que, quanto maior o número de parâmetros do modelo, menor o grau de liberdade do resíduo e maior a soma de quadrados do modelo de regressão. Draper \& Smith (1981) propuseram a seguinte fórmula:

$$
R_{\text {ajust }}^{2}=\frac{(n-1) R^{2}-p}{n-p-1},
$$

em que $\mathrm{R}^{2}$ ajust $=$ coeficiente de determinação ajustado; $\mathrm{p}=$ número de parâmetros do modelo; $\mathrm{n}$ = número de observações; e $\mathrm{R}^{2}=$ coeficiente de determinação.

Em razão das características de ajustamento, foi escolhida uma das funções para o teste da igualdade de parâmetros e da identidade de modelos de regressão não-linear (Regazzi, 2003). Este teste determina se uma única curva de lactação pode ser adequada para descrever as diferenças entre as lactações e entre as raças.

\section{Resultados e Discussão}

Foram encontrados baixos valores para o coeficiente de determinação ajustado na estimativa da curva de lactação 
(Tabela 2). Estes resultados podem ser explicados pela utilização de dados individuais na estimativa dos parâmetros, visto que alguns pesquisadores utilizam a média de produção por período, o que tende a eliminar a variação dos dados. Valores semelhantes foram encontrados por McManus et al. (1997), em estudo com vacas da raça Holandesa, no qual buscaram o ajuste da curva de lactação. Entretanto, foram inferiores aos encontrados por Ribeiro et al. (1997), para cabras mestiças. Os valores de $\mathrm{R}^{2}$ ajustado encontrados na literatura são, em sua maioria, próximos a $100 \%$, mesmo havendo enorme variação entre os valores observados e preditos (Regazzi, 2003). Segundo Ratkowsky (1990), o R ${ }^{2}$ não tem qualquer significado óbvio para modelos de regressão não-linear como único critério de seleção.

Os dados não se ajustaram aos modelos 2, 7 e 10, que apresentaram valores de MDA e QMR discrepantes, e, portanto, essas equações são inadequadas para o ajuste de curvas de lactação dos dados avaliados.

O modelo 6 , embora tenha apresentado bom ajuste numérico, não estimou satisfatoriamente o pico de produção na avaliação gráfica das curvas estimada e observada, semelhante ao que ocorreu com o modelo de Wood (1967), que, apesar de vastamente indicado e analisado na literatura (Kellogg et al., 1977; Madalena et al., 1979; Wood, 1980), não determinou satisfatoriamente o pico de produção.

De acordo com os critérios descritos na Tabela 2, os modelos que apresentaram melhor comportamento para o

Tabela 2 - Coeficiente de determinação ajustado $\left(R^{2}\right.$ ajustado), média dos desvios absolutos (MDA) e quadrado médio do resíduo (QMR) segundo os modelos

Table 2 - $\quad$ Adjusted coefficient of determination $\left(R^{2}{ }_{\text {adjusted }}\right)$, absolute mean deviation (AMD) and residual mean square (MSR) according to the models

\begin{tabular}{llccc}
\hline & $\begin{array}{l}\text { Função } \\
\text { Function }\end{array}$ & $\begin{array}{c}\mathrm{R}^{2} \text { ajustado } \\
R^{2} \text { adjusted }\end{array}$ & MDA & QMR \\
\hline 1 & $Y=a e^{b t}-a e^{-c t}$ & 0,391 & 0,087 & 0,949 \\
2 & $Y=a e^{\left(b t-c t^{2}\right)}$ & 0,386 & 1,588 & 0,953 \\
3 & $Y=t / a+b t+c t^{2}$ & 0,394 & 0,063 & 0,946 \\
4 & $Y=a t^{b} e^{-c t}$ & 0,389 & 0,102 & 0,951 \\
5 & $Y=\ln (a)+b \ln (t)-c t$ & 0,382 & 0,127 & 0,957 \\
6 & $Y=a+b t-c t^{2}$ & 0,387 & 0,076 & 0,953 \\
7 & $Y=a-b t-a e^{-c t}$ & 0,382 & 2,480 & 7,129 \\
8 & $Y=a-b t+c \ln (t)$ & 0,382 & 0,137 & 0,957 \\
9 & $Y=a t^{b c} e^{-c t}$ & 0,389 & 0,102 & 0,951 \\
10 & $Y=a t e^{-c t}$ & 0,267 & 1,847 & 1,225 \\
11 & $Y=a e^{\left(b t^{-}-c t^{\prime}+d / t\right)}$ & 0,395 & 0,050 & 0,945 \\
12 & $Y=a t^{b e(-c t)}$ & 0,378 & 0,100 & 0,960 \\
\hline
\end{tabular}
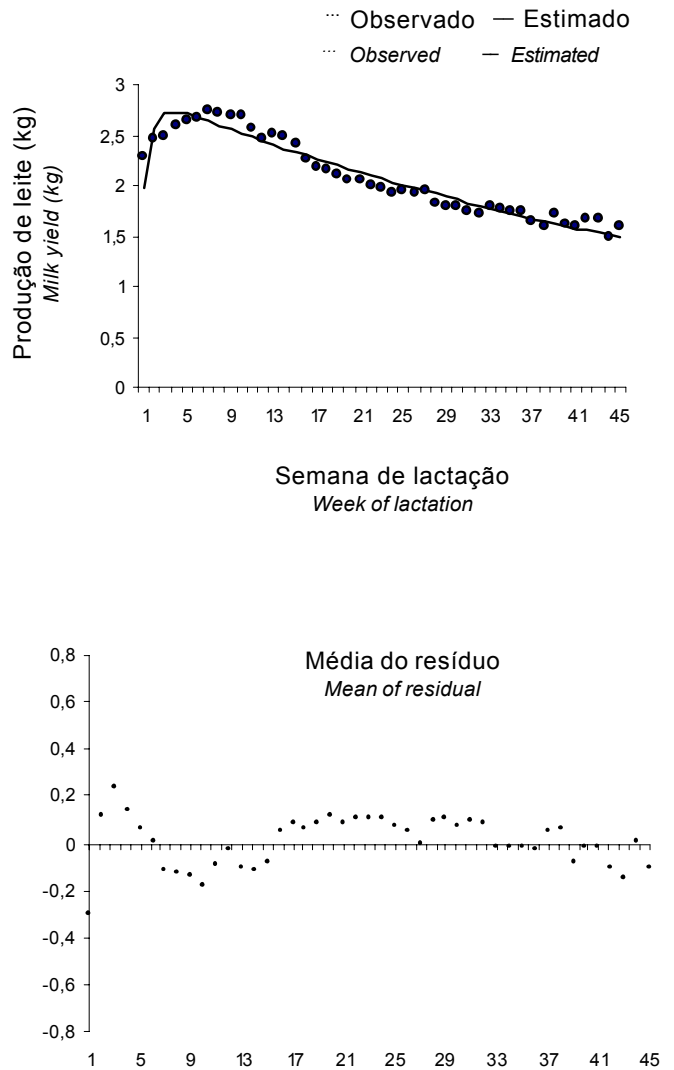

Figura 1 - Curvas de lactação estimada e observada e média do resíduo para o modelo 1 (Brody et al., 1924).

Figure 1 - Observed and estimated lactation curve and mean of residual for the model 1 (Brody et al., 1924).
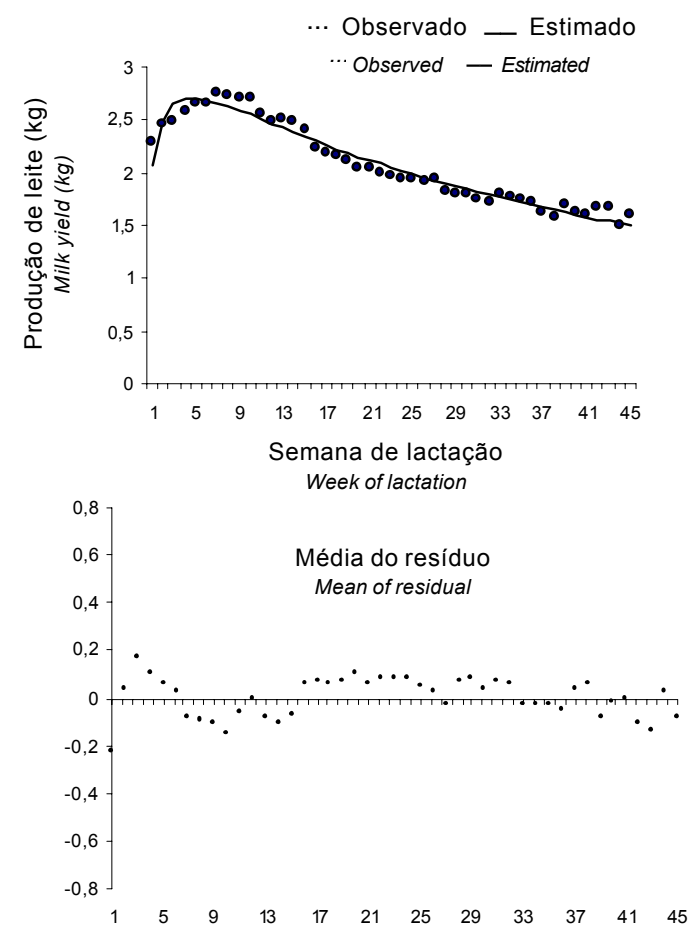

Figura 2 - Curvas de lactação estimada e observada e média do resíduo para o modelo 3 (Nelder, 1966).

Figure 2 - Observed and estimated lactation curve and mean of residual for the model 3 (Nelder, 1966). 

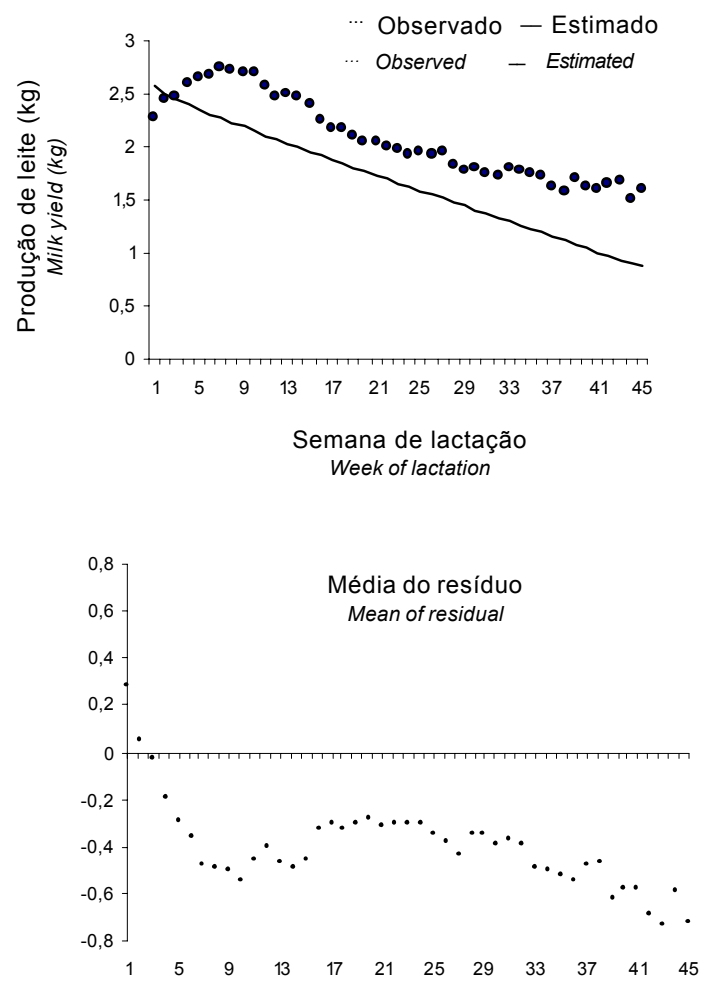

Figura 3 - Curvas de lactação estimada e observada e média do resíduo para o modelo 8 (Sing \& Gopal, 1982).

Figure 3 - Observed and estimated lactation curve and mean of residual for the model 8 (Singh \& Gopal, 1982).

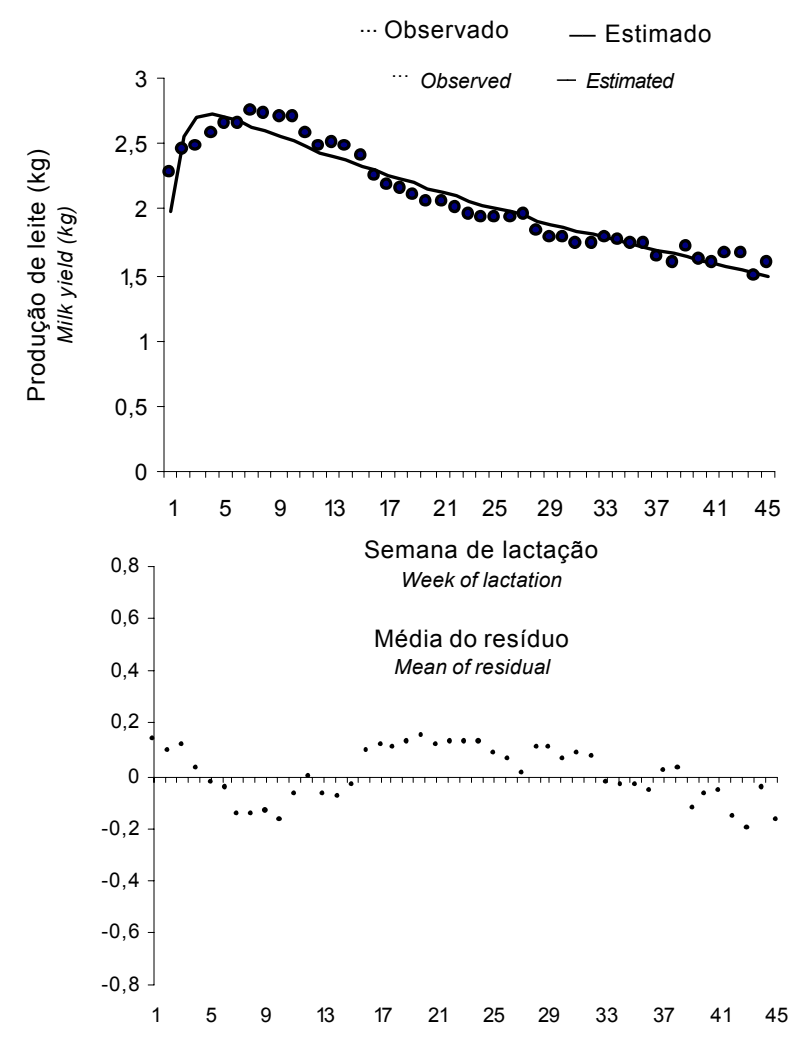

Figura 4 - Curvas de lactação estimada e observada e média do resíduo para o modelo 9 (Danhoa, 1981).

Figure 4 - Observed and estimated lactation curve and mean of residual for the model 9 (Danhoa, 1981).
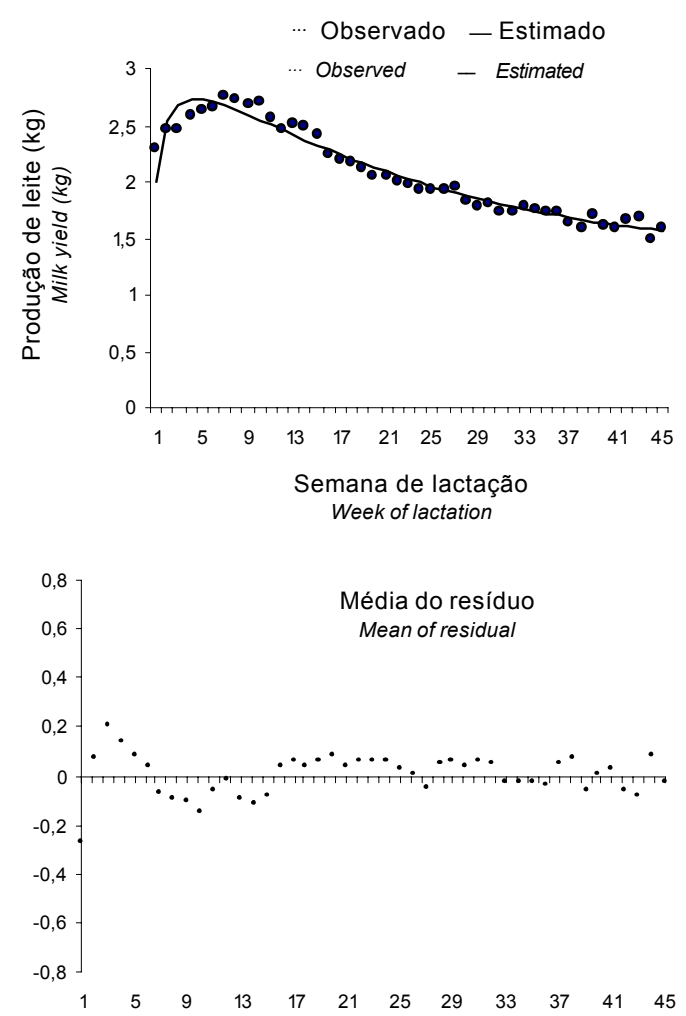

Figura 5 - Curvas de lactação estimada e observada e média do resíduo para o modelo 11 (Morant \& Gnanasakthy, 1989).

Figure 5 - Observed and estimated lactation curve and mean of residual for the model 11 (Morant \& Gnanasakthy, 1989).

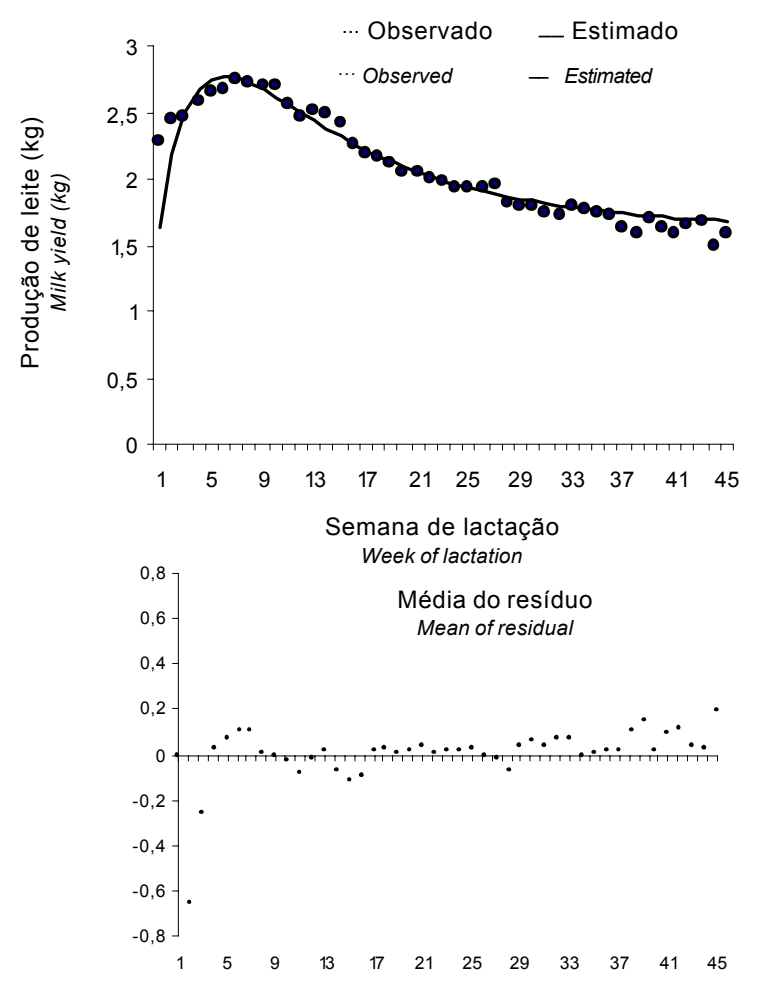

Figura 6 - Curvas de lactação estimada e observada e média do resíduo para o modelo 12 (Cappio-Borlino, 1995).

Figure 6 - Observed and estimated lactation curve and mean of residual for the model 12 (Cappio-Borlino, 1995). 
ajuste da curva média da produção de leite foram os modelos 1 (Brody et al., 1924), 3 (Nelder, 1966), 8 (Singh \& Gopal, 1982), 9 (Dhanoa, 1981), 11 (Morant \& Gnanasakthy, 1989) e 12 (Cappio-Borlino et al., 1995). Nas Figuras 1 a 4 constam os gráficos de dispersão das médias dos resíduos e as curvas estimada e observada para estes seis modelos.

O modelo 1 (Figura 1) apresentou pequenas variações na estimativa da fase de declínio da produção de leite, superestimando a produção em parte do tempo da lactação. Os modelos 3 e 11 (Figuras 2 e 3 , respectivamente) tiveram tendências semelhantes, com diferenças no valor e no tempo de pico de produção. O modelo 12 (Figura 4) foi aquele com melhor ajustamento para o valor e o tempo do pico de produção, entretanto, subestimou a fase inicial da lactação, incorrendo em maior MDA, comprovando a própria característica do modelo.

O modelo de Sing \& Gopal (1982), embora com bons valores de ajustamento (Tabela 2), não se mostrou adequado para a estimativa da curva de lactação, pela análise gráfica, pois não estimou o pico de produção e subestimou a produção de leite na maior parte da lactação.

O modelo de Danhoa (1981), por sua vez, apresentou problemas na estimativa da produção inicial, com valores superestimados, e no tempo para o pico de produção, que não foi adequado. Entretanto, após o pico de produção, esse modelo ajustou satisfatoriamente a curva de lactação.

O modelo 11 (Morant \& Gnanasakthy, 1989) proporcionou o melhor coeficiente de determinação, o menor resíduo médio e, conseqüentemente, o melhor ajuste para a produção média. Contudo, pareceu ser inadequado para estimação de curvas de lactação para cada animal, pois não propiciou bom ajuste da curva, como observado também por Andonov et al. (2000). Esse fato reforça a necessidade de se efetuar uma análise baseada em mais de um fator de comparação e não somente pelo $\mathrm{R}^{2}$ ou MDA, estimado apenas para a curva média.

O modelo proposto por Cappio-Borlino et al. (1995), modelo 12, também apresentou bom ajuste para a curva de lactação, mas subestimou significativamente a produção

Tabela 3 - Valores encontrados para os parâmetros das equações 1, 3, 11 e 12

\begin{tabular}{llcccl} 
Table 3 & $-\quad$ Values estimated for the parameters of equations $1,3,11,12$ \\
\hline & $\begin{array}{c}\text { Modelo } \\
\text { Model }\end{array}$ & \multicolumn{4}{c}{$\begin{array}{c}\text { Parâmetro } \\
\text { Parameter }\end{array}$} \\
\cline { 2 - 6 } & & $\mathrm{a}$ & $\mathrm{b}$ & $\mathrm{d}$ \\
\hline 1 & $Y=a e^{b t}-a e^{-c t}$ & 2,9272 & 0,0151 & 1,1798 & - \\
3 & $Y=t / a+b t+c t^{2}$ & 0,1873 & 0,2907 & 0,00825 & - \\
11 & $Y=a e^{\left(b t^{\prime}-c t^{\prime}+d / t\right)}$ & 7,8945 & 3,8113 & 2,1714 & $-0,5092$ \\
12 & $Y=a t^{b e(-c t)}$ & 1,6328 & 0,5094 & 0,0912 & - \\
\hline
\end{tabular}

inicial. Esse fato pode ser atribuído à característica do modelo, que, segundo os autores, tenta resolver o problema da estimação dos primeiros dias, quando existem poucos registros de informações.

Observam-se na Tabela 3 os valores estimados para os parâmetros, os quais possuem significados diferentes e interpretações distintas. Mesmo para os modelos que proporcionaram melhor ajuste, ocorreram variações na estimação, tanto no início quanto no final da lactação. Os resultados foram semelhantes aos encontrados por Ribeiro et al. (1997), que verificaram subestimação e superestimação para produção em diversos segmentos da curva.

Com base nas informações do gráfico de distribuição de resíduo, da média dos desvios absolutos e do quadrado médio dos resíduos e, principalmente, pela comparação das curvas estimada e observada por avaliação visual, os modelos de Brody et al. (1924) e de Nelder (1966) foram considerados os de melhor ajuste à curva de lactação do rebanho.

Apesar da eficiência dos dois modelos, a equação de Nelder (1966) foi escolhida para a realização do teste de identidade de modelos por ter sido bastante utilizada na estimativa de lactação para bovinos (Papajcsik \& Bodero, 1988).

Nessa função, o parâmetro $a$ é a taxa de crescimento da curva até o pico de produção; $b$, a inclinação média da curva de lactação; e $c$, a inclinação na fase de declínio. O pico de produção foi de 2,69 kg em 27 dias e o tempo para atingir o pico de produção foi semelhante ao encontrado por Cobby \& Le Du (1978) e Fernández et al. (2002).

Pelo teste de identidade de modelos (Tabela 4), verificou-se que os parâmetros $b$ e $c$ apresentaram valores diferenciados para as duas raças, comprovando que existem diferenças no formato da curva de produção entre as cabras Saanen e Alpina, não necessariamente implicando em diferenças produtivas. Também foram observadas diferenças entre os parâmetros de cada uma das quatro lactações

Tabela 4 - Resumo do teste de igualdade de parâmetros e identidade de modelos para a curva de lactação das raças Saanen e Alpina aplicado ao modelo 3 de Nelder (1966)

Table 4 - Summary of the test of equality of parameters and the identity of the models for the lactation curve of Saanen and Brown Alpine goats, applied to model 3 (Nelder, 1966)

\begin{tabular}{|c|c|c|c|}
\hline \multirow[t]{2}{*}{$\begin{array}{l}\text { Hipótese } \\
\text { Hypothesis }\end{array}$} & \multirow[t]{2}{*}{$\begin{array}{l}\text { GL } \\
D F\end{array}$} & \multicolumn{2}{|c|}{$\begin{array}{l}\text { Raça } \\
\text { Breed }\end{array}$} \\
\hline & & $\begin{array}{c}\chi_{\text {calculado }}^{2} \\
\chi_{\text {calculated }}^{2}\end{array}$ & $\begin{array}{c}\text { Valor- } \mathrm{p} \\
P \text { value } \\
\mathrm{P}\left(\chi_{\mathrm{v}}^{2}>\chi_{\text {calculado }}^{2}\right) \\
P\left(\chi_{v}^{2}>\chi_{\text {calculated }}^{2}\right)\end{array}$ \\
\hline $\mathrm{H}_{\mathrm{o}}: \mathrm{a}_{1}=\mathrm{a}_{2}=\mathrm{a}$ & 1 & 0 & $\mathrm{P}>0,05$ \\
\hline $\mathrm{H}_{\mathrm{o}}: \mathrm{b}_{1}=\mathrm{b}_{2}=\mathrm{b}$ & 1 & 17,48 & $\mathrm{P}<0,01$ \\
\hline $\mathrm{H}_{\mathrm{o}}: \mathrm{c}_{1}=\mathrm{c}_{2}=\mathrm{c}$ & 1 & 6,00 & $\mathrm{P}<0,01$ \\
\hline
\end{tabular}


Tabela 5 - Resumo do teste de igualdade de parâmetros e identidade de modelos para a curva de lactação de cabras, utilizando-se a ordem de lactação, aplicado ao modelo 3 (Nelder, 1966)

Table 5 - Summary of the test of equality of parameters and the identityof the models for the lactation curve, using order of lactation, applied to model 3 (Nelder, 1966)

\begin{tabular}{|c|c|c|c|}
\hline \multirow[t]{2}{*}{$\begin{array}{l}\text { Hipótese } \\
\text { Hypothesis }\end{array}$} & \multirow[t]{2}{*}{$\begin{array}{l}\text { GL } \\
D F\end{array}$} & \multicolumn{2}{|r|}{$\begin{array}{l}\text { Raça } \\
\text { Breed }\end{array}$} \\
\hline & & $\begin{array}{c}\chi_{\text {calculado }}^{2} \\
\chi_{\text {calculated }}^{2}\end{array}$ & $\begin{array}{c}\text { Valor- } \mathrm{p} \\
P \text { value } \\
\mathrm{P}\left(\chi^{2}{ }_{\mathrm{v}}>\chi_{\text {calculado }}^{2}\right) \\
P\left(\chi^{2}{ }_{v}>\chi_{\text {calculated }}^{2}\right)\end{array}$ \\
\hline $\mathrm{H}_{0}: \mathrm{a}_{1}=\mathrm{a}_{2}=\mathrm{a}_{3}=\mathrm{a}_{4}=\mathrm{a}$ & 1 & 5,034218 & $\mathrm{P}<0,05$ \\
\hline $\mathrm{H}_{\mathrm{o}}: \mathrm{b}_{1}=\mathrm{b}_{2}=\mathrm{b}_{3}=\mathrm{b}_{4}=\mathrm{b}$ & 1 & 33,14013 & $\mathrm{P}<0,01$ \\
\hline $\mathrm{H}_{\mathrm{o}}: \mathrm{c}_{1}=\mathrm{c}_{2}=\mathrm{c}_{3}=\mathrm{c}_{4}=\mathrm{c}$ & 1 & 318,2843 & $\mathrm{P}<0,01$ \\
\hline
\end{tabular}

(Tabela 5). Evidencia-se, portanto, que existem variações no formato da curva para as diferentes lactações estudadas, pois somente para o parâmetro $a$ não foi encontrada variação entre as raças.

Pelo mesmo modelo para cada uma das quatro lactações e das duas raças, os parâmetros $a, b$ e $c$ apresentaram valores diferentes em cada uma das lactações. De forma semelhante, quando avaliadas as raças, foram encontradas alterações para os parâmetros $b$ e $c$, comprovando a existência de variações no comportamento produtivo dos animais, configurando uma curva de lactação diferente para cada uma das quatro lactações e das duas raças. À medida que o animal avança em sua vida produtiva, ocorre um aumento gradativo da produção que determina um perfil de produção diferente da lactação anterior e, certamente, da seguinte. Para o parâmetro raça, a diferença no formato da curva para a mesma lactação representa as variações nas características biológicas e produtivas de cada raça, que podem ser atribuídas ao tempo de pico de produção ou, até mesmo, da persistência da lactação. Esta análise apenas evidencia as variações existentes em cada fase da vida produtiva (número de lactações do animal) ou em cada raça, as quais, em termos práticos, são desconsideradas para facilitar o estudo.

Constam na Tabela 6 as correlações encontradas para a produção de leite e para os parâmetros do modelo 3 .

A correlação foi média e negativa entre o parâmetro $c \mathrm{e}$ a produção de leite, indicando que, quanto menor a inclinação da curva na fase final da lactação (após o pico de produção), maior a produção de leite. Esse parâmetro está diretamente relacionado à persistência da lactação, visto que a redução da taxa de declínio após o pico provoca o prolongamento do período produtivo do animal. Assim, quanto maior a produção de leite, menor o decréscimo na produção nos estádios finais da lactação (Batra et al., 1987).
Tabela 6 - Correlações de Pearson entre os parâmetros da curva de lactação do modelo 3 e a produção total de leite

Table 6 - Pearson correlations between the estimated parameters for model 3 and total milk yield

\begin{tabular}{|c|c|c|c|c|}
\hline & $\begin{array}{c}\mathrm{PLT} \\
T M Y\end{array}$ & $\mathrm{a}$ & $\mathrm{b}$ & $\mathrm{c}$ \\
\hline P L T & 1 & $-0,11$ & $-0,20$ & $-0,59$ \\
\hline \multicolumn{5}{|l|}{$T M Y$} \\
\hline $\mathrm{a}$ & & 1 & $-0,37$ & 0,15 \\
\hline $\mathrm{b}$ & & & 1 & $-0,28$ \\
\hline $\mathrm{c}$ & & & & 1 \\
\hline
\end{tabular}

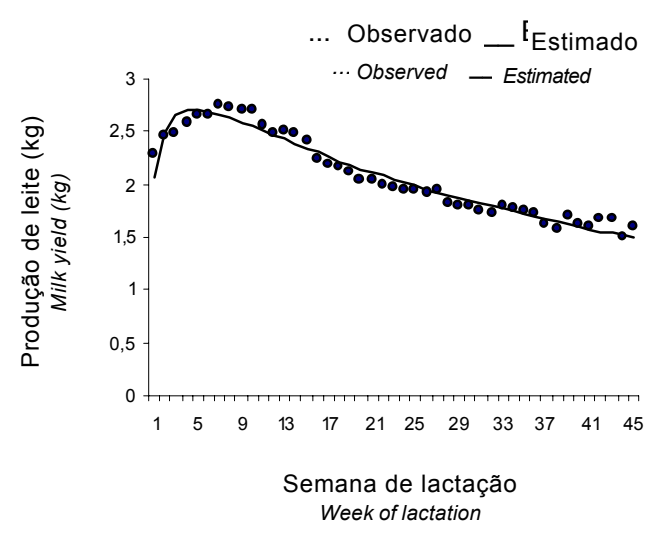

Figura 7 - Curvas de lactação estimada e observada para o modelo de Nelder (1966).

Figure 7 - Observed and estimated lactation curve for Nelder (1966) model.

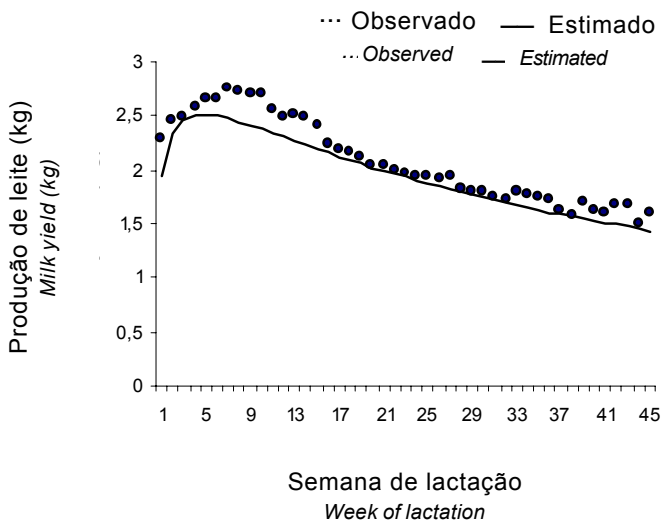

Figura 8 - Curvas de lactação estimada e observada para o modelo de Nelder (1966) com um aumento de $10 \%$ no valor do parâmetro $b$, mantendo a e $c$ constantes.

Figure 8 - Observed and estimated lactation curve for Nelder (1966) model increasing $10 \%$ in the value o of " $b$ " parameter, keeping "a" and "c" cosntants. 


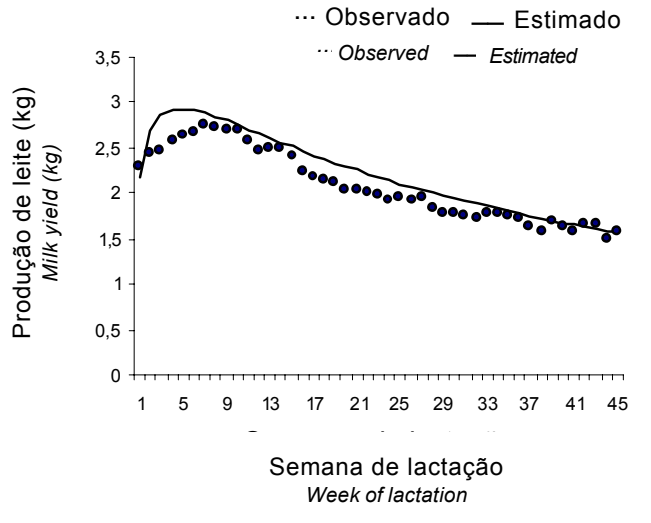

Figura 9 - Curvas de lactação estimada e observada para o modelo de Nelder (1966) com uma redução de $10 \%$ no valor do parâmetro $\mathrm{b}$, mantendo a e c constantes.

Figure 9 - Observed and estimated lactation curve for Nelder (1966) model decreasing $10 \%$ in the value o of "b" parameter, keeping "a" and "c" cosntants.

A correlação encontrada entre o parâmetro $b$ e a produção total de leite indica que o pico de produção é menos pronunciado à medida que $b$ aumenta, não influenciando o tempo para o pico de produção. Houve correlação negativa entre os parâmetros $b$ e $c$, sugerindo que animais com maior persistência tendem a apresentar pico de lactação menos pronunciado.

Essa diferença pode ser visualizada nas Figuras 7 a 9, em que foram demonstrados um aumento e uma redução de $10 \%$ no parâmetro $b$ para que se pudesse perceber melhor esta relação.

O parâmetro $a$ teve correlação negativa com a produção total de leite. Apesar da baixa magnitude, esse resultado pode indicar que a taxa de crescimento até o pico possui alguma influência sobre a PLT. Entretanto, foi negativamente correlacionado a $b$, indicando que uma seleção para rápido aumento da produção de leite e da taxa de crescimento até o pico implicará menor inclinação média da curva. O parâmetro $c$ teve correlação pequena e positiva $(0,15)$, sugerindo que a persistência esteja fracamente relacionada à taxa de crescimento da produção até o pico da lactação.

\section{Conclusões}

Para a comparação dos modelos, foi fundamental a observação visual das curvas observadas e estimadas, uma vez que algumas equações apresentaram valores aceitáveis para as características avaliadas, mas com falsa indicação de ajuste.

Foram observadas variações entre os parâmetros do modelo escolhido (Nelder, 1966) para as diferentes lactações e raças, comprovando a existência de diferenças na forma da curva de lactação para essas variáveis.
A maior correlação entre o parâmetro $c$ do modelo e a produção de leite indica que a produção dos animais é fortemente influenciada pela persistência da lactação.

\section{Literatura Citada}

ANDONOV, S.; DZABIRSKI, V.; SRBINOVSKA, S. Selection of dairy goats by using lactation curve model, In: INTERNATIONAL CONFERENCE ON GOATS, 7., Tours, 2000. Proceedings... Ivry-sur-seine: Institut de L'Elevage et INRA, 2000. v.2, p1049.

BATRA, T.R.; LIN, C.Y.; McALLISTER, A.J. Multitrait estimation of genetic parameters of lactation curves in Holstein heifers. Journal of Dairy Science, v.70, p.2105-2111, 1987.

BRODY, S.; RAGSDALE, A.C.; TURNER, C.W. The relation between the initial rise and the subsequent decline of milk secretion following parturition. The Journal of General Physiology, v.6, p.541-545, 1924.

BUENO, J.H.; OLIVEIRA, A.I.G.; CONÇALVES, T.M. et al. Estudo da curva de lactação em rebanhos da raça Holandesa no estado de Minas Gerais. In: REUNIÃO ANUAL DA SOCIEDADE BRASILEIRA DE ZOOTECNIA, 35., 1998, Botucatu. Anais... Botucatu: Sociedade Brasileira de Zootecnia,1998.

CAPPIO-BORLINO, A.; PULINA, G.; ROSSI, G. A non-linear modification of Wood's equation fitted to lactation curves of Sardinian dairy ewes, Small Ruminant Research, v.18, p.75$79,1995$.

CASOLI, C.; DURANTI, E.; MORBIDINI, L. et al. Quantitative and compositional variations of Massese sheep milk by parity and stage of lactation. Small Ruminant Research, v.2, p.47$62,1989$.

COBBY, J.M.; LE DU, Y.L.P. On fitting curves to lactation data. Animal Production, v.26, n.2, p.127-133, 1978.

COBUCI, J.A.; EUCLYDES, R.F.; VERNEQUE, R.S. et al. Curva de lactação na raça Guzerá, Revista Brasileira de Zootecnia, v.29, n.5, p.1332-1339, 2000.

DAVE, B.K. First lactation curve of Indian water buffalo. Jawaharlal Nehru Krishi Vishwa Vidyalaya Research Journal, v.5, p.93, 1971.

DHANOA, M.S. A note on an alternative form of the lactation model of wood. Animal Production, v.32, p.342, 1981.

DRAPER, N.M.; SMITH, H. Applied regression analysis. 2.ed. New York: Wiley, 1981.709p.

FERNÁNDEZ, C.; SANCHEZ, A.; GARCÉS, C. Modeling the lactation curve for test-day milk yield in murciano-granadina goats. Small Ruminant Research, v.46, p.29-41, 2002.

FREITAS, M.A.R.; FARO, L.; GADINI, C.H. Estudo da persistência da lactação de vacas da raça Holandesa. In: REUNIÃO ANUAL DA SOCIEDADE BRASILEIRA DE ZOOTECNIA, 34., 1997, Juiz de Fora. Anais... Juiz de Fora: Sociedade Brasileira de Zootecnia, 1997.

FUERTES, J.A.; GONZALO, C.; CARRIEDO, J.A. et al. Parametres of test day milk yield and milk componentes for dairy ewes, Journal of Dairy Science, v.81, p.1300-1307, 1998.

GIPSON, T. A.; GROSSMAN, M. Diphasic Analysis of lactation curves in dairy goats. Journal of Dairy Science, v.72, p.1035$1044,1989$.

KELlOGG, D.W.; URQUHART, N.S.; ORTEGA, A.J. Estimating Holstein lactation curves with a gamma curve. Journal of Dairy Science, v.60, n.8, p.1308-1315, 1977.

MADALENA, F.E.; MARTINEZ, M.L.; FREITAS, A.F. Lactation curves of Hostein-Friesian and Holstein-Friesian x Gir cows. Animal Production, v.29, p.101-107, 1979.

MCMANUS, C.; GUTH, T.L.F.; SAUERESSIG, M.G. Curvas de lactação em gado Holandês em confinamento total no DF. In: REUNIÃO ANUAL DA SOCIEDADE BRASILEIRA DE 
ZOOTECNIA, 34., 1997, Juiz de Fora. Anais... Juiz de Fora: Sociedade Brasileira de Zootecnia, 1997. p.74-76.

MORANT, S.V.; GNANASAKTHY, A. A new approach to the mathematical formulation of lactations curves. Animal Production, v.49, p.151-162, 1989.

NELDER, J.A. Inverse polynomials a useful group of multi-factor response functions. Biometrics, v.22, n.1, p.128-141, 1966.

OLIVEIRA, S.M.P. Análise de lactação de cabras $1 / 2$ pardoParda Alpina x Moxotó. Belo Horizonte: Universidade Federal de Minas Gerais, 1999. 65p. Tese (Doutorado em Genética e Melhoramento de Animais Domésticos) - Universidade Federal de Minas Gerais, 1999.

PAPAJCSIK, I.A.; BODERO, J. Modeling laactation curves of Friesian cow in a subtropical climate. Animal Production, v.47, n.2, p.201-207, 1988.

RATKOWSKY, D.A. Handbook of nonlinear regression models. New York: M. Dekker, 1990. 241p.

REGAZZI, A.J. Teste para verificar a igualdade de parâmetros e a identidade de modelos de regressão não-linear. Revista Ceres, v.50, n.287, p.9-26, 2003.

RIBEIRO, M.N.; ALBUQUERQUE, L.G.; PIMENTA FILHO, E.C. Comparação de funções matemáticas no ajuste da curva de lactação de cabras mestiças no Cariri paraibano. In: REUNIÃO ANUAL DA SOCIEDADE BRASILEIRA DE ZOOTECNIA, 34., 1997, Juiz de Fora. Anais... Juiz de Fora: Sociedade Brasileira de Zootecnia, 1997. p.272-274.

SARMENTO, J.L.R.; TORRES, L.A.; SOUZA, W.H. et al. Comparação de modelos de regressão não-linear no ajuste dacurva de crescimento de ovinos santa Inês In.: REUNIÃO ANUAL DA SOCIEDADE BRASILEIRA DE ZOOTECNIA, 40., 2003, Santa Maria. Anais... Santa Maria: Sociedade Brasileira de Zootecnia, 2003.
STATISTICAL ANALYSIS SYSTEM- SAS. User's guide: Statistics. version 8.0 Cary: 1999.

SHANKS, R.D.; BERGER, P.J.; FREEMAN, A.E. et al. Genetic aspects fo lactation curves. Journal of Dairy Science, v.64, p.1852-1860, 1981 .

SHERCHAND, L.; McNEW, R.W.; KELLOGG, D.W. et al. Selection of a mathematical model to generate lactation curves using daily milk yields of Holstein cows, Journal of Dairy Science, v.78, p.2507-2513, 1995.

SIKKA, L.C. A study of lactation as affected by buredity and environment. Journal Dairy Research, v.17, n.3, p.231-252, 1950 .

SINGH, R.P.; GOPAL, R. Lactation curves analysis of buffaloes maintained under village conditions. Indian Journal of Animal Science, v.52, n.12, p.1157-1160, 1982.

VARGAS, B.; KOOPS, W.J.; HERRERO, M. et al. Modeling extended lactations of dairy cows. Journal of Dairy Science, v.83, p.1371-1380, 2000.

WOOD, P.D.P. A note on the lactation curves of some high yielding British Friesian cows. Animal Production, v.30, n.2, p.299$302,1980$.

WOOD, P.D.P. Algebraic model of the lactation curve in cattle. Nature, v.206, p.164-165, 1967. 